\title{
Long-term Follow-up of Extensive Peri-anchor (Poly-L/D-lactic Acid) Cyst Formation after Arthroscopic Rotator Cuff Repair: A Case Report
}

\author{
Jong-Ho Kim, Jong-Ick Kim, Hyo-Jin Lee², Dong-Jin Kim², Gwang Young Sung², Dong-Ho Kwak², \\ Yang-Soo Kim ${ }^{2 \varpi}$
}

Department of Orthopedic Surgery, Wonkwang University Sanbon Hospital, Wonkwang University of School of Medicine, Gunpo, ${ }^{1}$ Department of Orthopedic Surgery, Incheon St. Mary's Will Hospital, Incheon, ${ }^{2}$ Department of Orthopedic Surgery, Seoul St. Mary's Hospital, College of Medicine, The Catholic University of Korea, Seoul, Korea

\begin{abstract}
Suture anchors are commonly used in shoulder surgeries, especially for rotator cuff tears. Peri-anchor cyst formation, however, is sometimes detected on follow-up radiologic image after surgery. The purpose of this report is to discuss the case of a patient who presented with regression of extensive peri-anchor cyst on postoperative 4-year follow-up magnetic resonance imaging and had good clinical outcome despite peri-anchor cyst formation after arthroscopic rotator cuff repair.
\end{abstract}

(Clin Shoulder Elbow 2019;22(2):100-105)

Key Words: Arthroscopy; Rotator cuff; Bioabsorbable anchor; Peri-anchor cyst; Degradation

Suture anchors are commonly used for shoulder surgery in cases of rotator cuff tear, instability, and fracture. First-generation suture anchors were metal and provided excellent fixation but had a high risk of failure, including suture loosening and anchor migration that resulted in cartilage damage. In addition, postoperative follow-up magnetic resonance imagings (MRIs) were often distorted because of the metallic artifact effect. To overcome the disadvantages of metal anchors, second-generation anchors were produced as bioabsorbable screws. Polyglycolic acid (PGA) was used as the first bioabsorbable material for the new anchors. However, due to the rapid degradation and loss of strength of PGA in early postoperative stages, use of this material was soon discontinued. Polylactic acid enantiomers (PLLA), which degrade more slowly, were subsequently introduced. In addition, to control the rate of degradation, copolymers of monomers, such as poly-L/D-lactic acid (PLDLA) and PLLA with PGA, have been used. $^{1)}$

Peri-anchor cyst formation is sometimes detected on followup radiographs and MRI after arthroscopic shoulder surgery.
However, the correlation between peri-anchor cyst formation and clinical outcome remains unclear. Nho et al. ${ }^{2)}$ suggested that glenohumeral synovitis, glenoid osteolysis, loose bodies, and chondral injury are complications related to peri-anchor cyst. Kim et al. ${ }^{1)}$ reported that almost one-half of patients (46.4\%) had fluid collection around the suture anchors approximately 1 year after arthroscopic rotator cuff repair (ARCR), and 19.1\% of those patients had significant fluid collection. However, there was not a significant correlation between the grade of fluid collection and the integrity of the repair. ${ }^{1)}$

In this report, we discuss the case of a patient who had an extensive peri-anchor cyst that regressed in size and had good shoulder function with intact integrity of the repaired rotator cuff at 4 years postoperative ARCR.

\section{Case Report}

A 65-year-old male visited Seoul St. Mary's Hospital complaining of right shoulder pain over the course of 6 months.

Received October 29, 2018. Revised December 18, 2018. Accepted December 23, 2018.

Correspondence to: Yang-Soo Kim

Department of Orthopedic Surgery, The Catholic University of Korea, Seoul St. Mary's Hospital, 222 Banpo-daero, Seocho-gu, Seoul 06591, Korea

Tel: +82-2-2258-6117, Fax: +82-2-535-9834, E-mail: kysoos@catholic.ac.kr, ORCID: https://orcid.org/0000-0003-4267-7880

IRB approval: The Catholic University of Korea, St. Mary's Hospital (No.: KC17OESI0118).

Financial support: None. Conflict of interests: None. 
He had a history of hypertension and atrial fibrillation. He had experienced an episode of transient ischemic attack in the brain but had no sequalae of brain damage. He complained of a painful arc during forward flexion of his right shoulder, but the shoulder had nearly full range of motion. Rent sign was positive and manual muscle power testing of shoulder flexion was scored as grade 4 . Tests for the impingement sign and speed were positive, which suggested the presence of subacromial bursitis and pathology of the long head of the biceps tendon. On MRI, a $2 \mathrm{~cm}$ medially retracted and anteroposterior $2.5 \mathrm{~cm}$ sized fullthickness tear of the supraspinatus tendon was noted (Fig. 1A). No bone abnormalities were observed (Fig. 2A).

The patient underwent ARCR. During the operation, an ap- proximately $2.0 \times 2.5 \mathrm{~cm}$ full-thickness tear of the supraspinatus tendon was found. Partial tears of the long head of the biceps tendon and the subscapularis tendon were also noted. We repaired the supraspinatus tendon with the transosseous-equivalent repair technique using two Bio-Paladin anchors $(5.0 \mathrm{~mm}$; CONMED, Utica, NY, USA) for medial row fixation and two Pop-Locks (3.5 mm; CONMED) for lateral row fixation (Fig. 3A). Tenodesis of the long head of the biceps tendon was performed using a Bio-Swivelock (5.5 mm; Arthrex, Naples, FL, USA). The subscapularis tendon was repaired with a Bio-Swivelock stitch for tenodesis of the long head of the biceps.

One month post-surgery, the patient started pendulum exercises and a rehabilitation program. At two months post-oper-
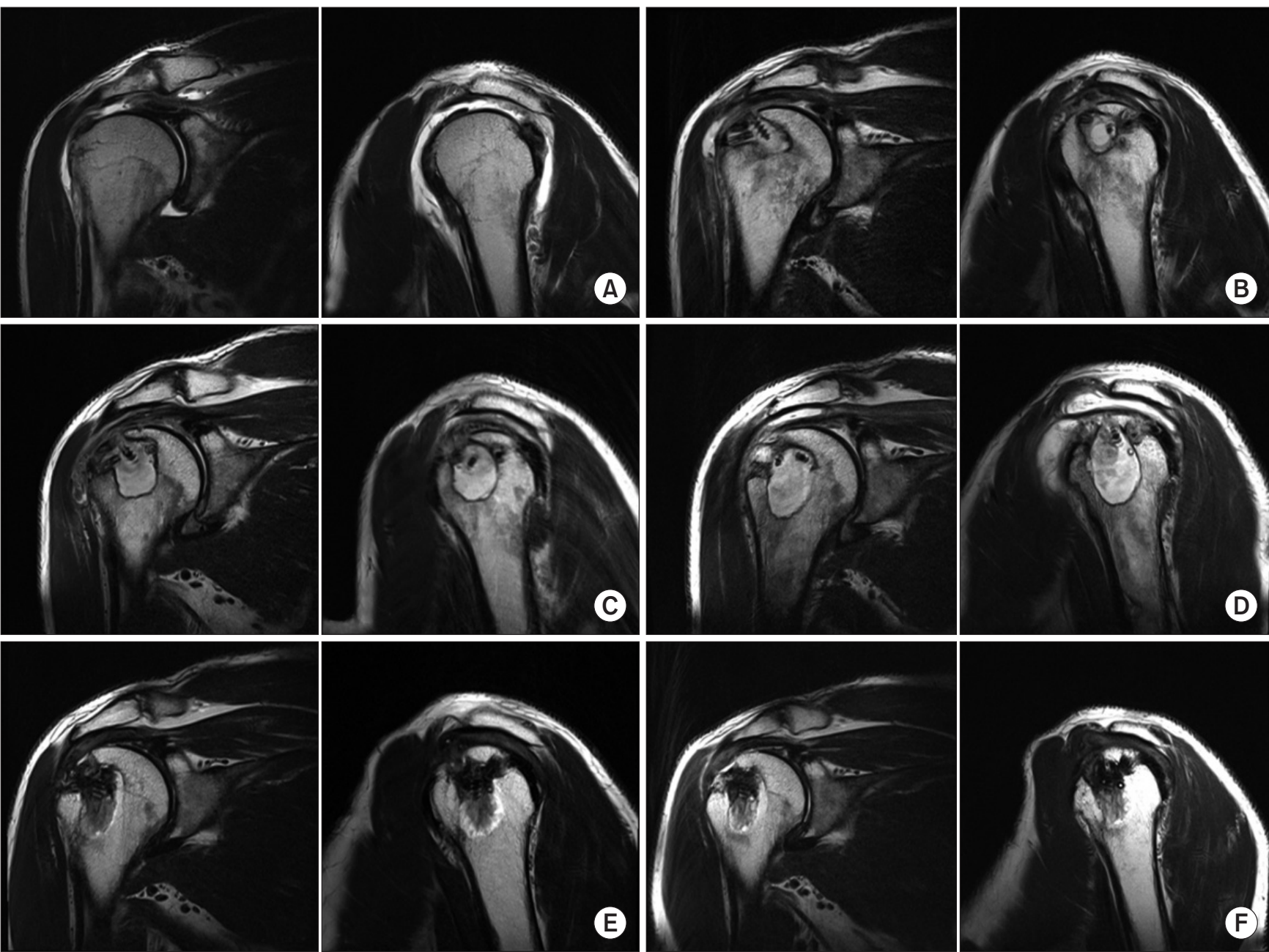

Fig. 1. Serial follow-up magnetic resonance imagings (MRIs). (A) Initial preoperative MRI, T2-weighted coronal and sagittal images. A full-thickness tear of the supraspinatus tendon was observed with a medio-lateral retraction diameter of $2 \mathrm{~cm}$. (B) Postoperative 6-month follow-up MRI shows an approximately $18 \times 16 \times 14 \mathrm{~mm}$ peri-anchor cyst at the medial anchor within the humeral head. (C) A follow-up MRI at 1 year after surgery shows an increase in the size of the cystic lesion $(25 \times 18 \times 18 \mathrm{~mm})$ compared to the postoperative 6-month follow-up MRI. However, the continuity of the repaired cuff was well-maintained. (D) A follow-up MRI at 20 months after surgery shows extensive enlargement of the cyst $(37 \times 22 \times 22 \mathrm{~mm})$ and a change in the inclination of the medial anchor. A sagittal image shows substantial subacromial and subdeltoid effusion. (E) A follow-up MRI at 3 years post-surgery shows granulation tissue filling the cavity and apparently fused with the anchor. The integrity of the repaired rotator cuff was well-maintained. The subacromial effusion was reduced, and the size of the perianchor cyst had decreased slightly $(35 \times 23 \times 18 \mathrm{~mm})$. (F) A follow-up MRI at 4 years post-surgery shows decreased size of the peri-anchor cyst lesion $(30 \times 20 \times 15$ $\mathrm{mm})$. Granulation tissue was fused to the anchor and filled the cavity. The continuity of the repaired tendon was well-maintained. 

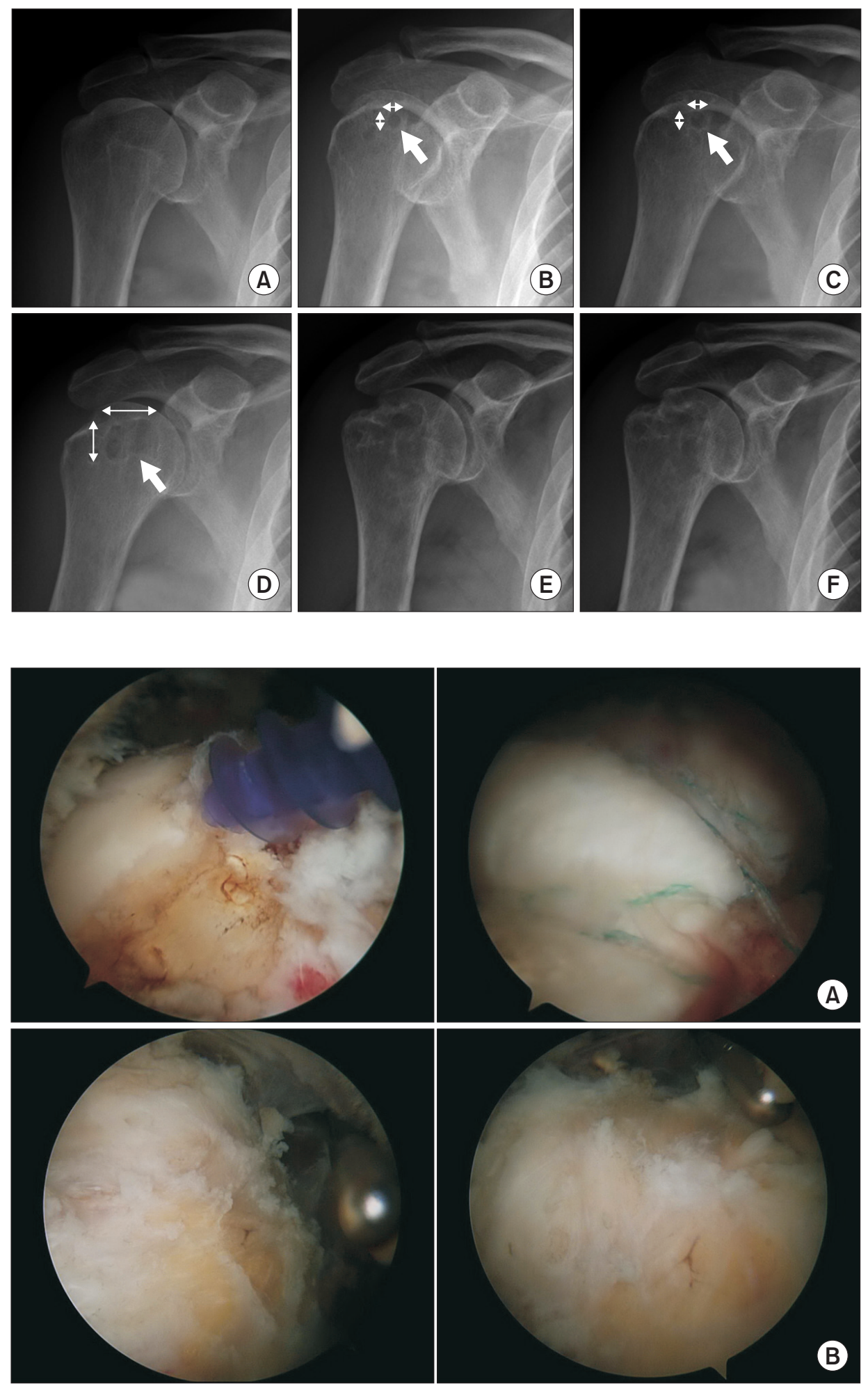

Fig. 2. Serial follow-up X-rays. The arrows indicate the cyst. The left-right arrows indicate the diameter of the cyst. (A) No bone abnormalities were noted on the preoperative X-ray. (B) A postoperative 3-month follow-up X-ray. An approximately $5 \times 5 \mathrm{~mm}$ cyst was observed at the medial anchor site on the humeral head. (C) A postoperative 6-month follow-up X-ray. The size of cyst was slightly increased to $7 \times 7 \mathrm{~mm}$. (D) A postoperative 20 -month follow-up X-ray. The size of cyst was significantly increased. Postoperative 3-year (E) and 4-year (F) Xrays show regression of the peri-anchor cyst.
Fig. 3. Intraoperative arthroscopic findings. (A) A 5.0-mm Paladin anchor (Bioabsorbable; CONMED, Utica, NY, USA) was used for medial row fixation. Rotator cuff repair was performed using the transosseous equivalent repair technique. (B) View from the additional arthroscopy performed at 4 years and 2 months after the primary surgery. Arthroscopy shows that the continuity of the repaired cuff is well-maintained. ation, the Sleeper's stretching exercise and the pulley exercise were prescribed. At three months post-operation, rubber exercises on the shoulder were begun in order to improve external rotation power. On the 3-month post-operative follow-up X-ray, an approximately $5 \times 5 \mathrm{~mm}$ cyst was observed on the medial an- chor site of the humeral head (Fig. 2B). The patient complained of mild pain during forward flexion and internal rotation, but there was no aggravation of pain compared with the previous follow-up visit. Rehabilitation exercises were continued until the next follow-up visit. Six months post-surgery, a follow-up X- 
ray showed that the cyst had increased in size to $7 \times 7 \mathrm{~mm}$ (Fig. 2C). In addition, MRI showed an approximately $18 \times 16 \times 14$ $\mathrm{mm}$ peri-anchor cyst around the medial anchor within the humeral head and a sub-deltoid effusion on the lateral side of the humeral head (Fig. 1B). Corticosteroid (triamcinolone, subacromial space $20 \mathrm{mg}+$ glenohumeral joint $20 \mathrm{mg}$ ) was injected via sonographic guidance to relieve pain and improve the range of motion. At one year post-surgery, follow-up MRI showed that the size of the cystic lesion had increased to $25 \times 18 \times 18 \mathrm{~mm}$, although the continuity of the repaired cuff was well-maintained (Fig. 1C). Pain in the repaired shoulder was reduced, and functional scores were improved (Table 1).

Twenty months post-surgery, the patient complained of severe pain in his right shoulder with no specific cause. MRI showed extensive enlargement of the cyst and a change in the inclination of the medial anchor, which appeared to be floating. Nonetheless, the continuity of the repaired rotator cuff was maintained (Fig. 1D). The amount of subacromial effusion had increased; therefore, aspiration of the subacromial effusion was performed under sonographic guidance (Fig. 4), and subacromial corticosteroid injection (triamcinolone, $40 \mathrm{mg}$ ) was administered. Cytological analysis indicated the presence of neutrophils and a few macrophages, but there were no infectious microbes.

A follow-up MRI was performed 3 years after the operation. On a T2-weighted image, low-signal intensity tissue was observed at the cystic lesion, and the integrity of the repaired rotator cuff was well-maintained. Also, subacromial effusion was reduced and the continuity of the repaired cuff was maintained (Fig. 1E). Four years post-surgery, the patient visited our clinic for routine follow-up and did not complain of pain, limited range of motion, or weakness. The final follow-up MRI showed some granulation tissues that were fused with the anchors and had filled the cavity. The continuity of the repaired tendon was well- maintained (Fig. 1F).

Four years and 3 months after the surgery, the patient underwent a procedure for cubital tunnel syndrome at the ipsilateral elbow. After receiving the patient's consent, a second arthroscopic procedure was performed on the right shoulder, which revealed that the integrity of the repaired rotator cuff was well-maintained and that some fibrotic tissues and bursa were comingled above the repaired cuff (Fig. 3B).

\section{Discussion}

The advantage of bio-absorbable suture anchors is that they are degraded and absorbed over time, thereby theoretically

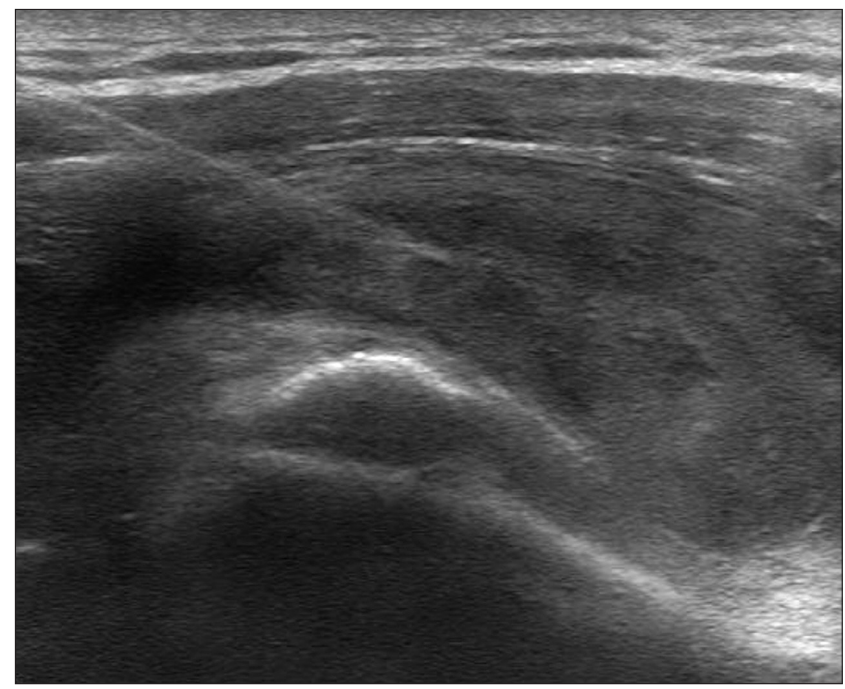

Fig. 4. An ultrasonographic image at 20 months after surgery. Aspiration of subacromial effusion and corticosteroid injection were performed under sonographic guidance.

Table 1. Postoperative Follow-up Range of Motion and Functional Scores of the Patient's Right Shoulder

\begin{tabular}{|c|c|c|c|c|c|c|}
\hline \multirow{2}{*}{ Variable } & \multicolumn{6}{|c|}{ Postoperative follow-up period (mo) } \\
\hline & 3 & 6 & 12 & 20 & 36 & 48 \\
\hline \multicolumn{7}{|l|}{ Range of motion } \\
\hline Forward flexion $\left(^{\circ}\right)$ & 110 & 130 & 140 & 130 & 130 & 140 \\
\hline Abduction external rotation $\left(^{\circ}\right)$ & 30 & 40 & 80 & 70 & 70 & 80 \\
\hline Side external rotation $\left(^{\circ}\right)$ & 30 & 60 & 80 & 80 & 80 & 80 \\
\hline Internal rotation & $\mathrm{L} 45$ & L1 & $\mathrm{TL}$ & $\mathrm{L} 2$ & $\mathrm{~T} 10$ & T8 \\
\hline \multicolumn{7}{|l|}{ Functional score } \\
\hline VAS pain & 5 & 5 & 3 & 7 & 2 & 1 \\
\hline ASES & 62 & 64 & 82 & 56 & 85 & 96 \\
\hline Constant score & 52 & 58 & 85 & 50 & 80 & 95 \\
\hline Korean shoulder score & 54 & 60 & 82 & 52 & 82 & 96 \\
\hline
\end{tabular}

VAS: visual analog scale, ASES: American Shoulder and Elbow Surgeons. 
avoiding both the complications of metallic anchors and interference in revision surgery. They are also generally radiolucent and thus do not interfere with imaging. ${ }^{3{ }^{3}}$ In addition, they are as strong as metallic suture anchors in secure tendon-to-bone repair. ${ }^{4)}$

Speer and Warren ${ }^{5)}$ emphasized several requirements of bioabsorbable implants that are used during shoulder arthroscopy. The implant must have initial fixation strength in order to coapt soft tissues to bone, and both the material property and the degradation time of the implant must be sufficiently long to allow the tissue to heal and regain mechanical integrity. However, the implant cannot degrade too slowly in order to avoid complications. The implant material cannot cause toxicity, antigenicity, pyogenicity, or carcinogenicity. Metallic anchors and first-generation PGA anchors did not satisfy these requirements. ${ }^{1)}$

Bio-Paladin anchors (5.0 mm; CONMED), which were used in the current case, are composed of poly-96L/4D-lactide copolymers. Kim et al. ${ }^{1)}$ reported that the prevalence of perianchor cysts at second-generation PLDLA anchors, like BioPaladin anchors, is $51.0 \%$. However, they observed no statistical difference in the healing rate between patients with and without cyst formation. In this case, the integrity of the repaired rotator cuff was well-maintained and there was good shoulder function at the final follow-up. We propose that the formation of a peri-anchor cyst with second-generation anchors, like PLDLA anchors, is not directly related to the outcome of ARCR.

Bio-absorbable suture anchors go through five stages after insertion into bone. ${ }^{6}$ In stage 1, water is absorbed into the rotator cuff anchor from the surrounding environment. In stage 2, the polymer in the rotator cuff anchor undergoes hydrolysis, resulting in decreased holding strength. In stage 3, the rotator cuff anchor fragments and absorption begins, which results in a decrease in fixation strength. In stages 4 and 5, the implant fragments are phagocytized, and the products enter the Krebs cycle and are eliminated through respiration. The entire process of bio-absorption takes between 5 months and 2 years. SaikkuBäckström et al. ${ }^{7)}$ used intramedullary nails of poly-96L/4Dlactide co-polymers for internal fixation of osteotomies of the femoral diaphysis in 43 adult rabbits. Soon after fixation, the implant was surrounded by a thin sheath of connective tissue; after 12 weeks, the implant was surrounded by bone. The implant slowly disintegrated, and the polymer debris was phagocytosed by macrophages. The implant had disappeared within 3 years. Using MRI, Macarini et al. ${ }^{8)}$ studied the degradation of PLDLA interference screws over the course of 3 years after surgery. In 34 of 35 patients, the screw eventually degraded and was not detectable. The authors noted cyst-like formations during the screw degradation process. Barber and Dockery ${ }^{9}$ reported that PLLA was completely absorbed within 7 years when used in $\mathrm{ACL}$ reconstruction. When complete reabsorption was observed, screws were not replaced with bone but rather with partially cal- cified fibrous tissue.

In the current case, peri-anchor cyst formation was first detected by X-ray at 3 months postoperation. The size of the peri-anchor cyst increased until 20 months post-operation, but the continuity of the repaired rotator cuff tendon was wellmaintained. Rodeo et al. ${ }^{10)}$ proposed a tendon-to-bone healing sequence from their studies in a canine model. At 12 weeks, collagen fibers resembling Sharpey's fibers connected the tendon to the surrounding bone and aligned in the direction of the pull of the musculotendinous unit. The continuity of the collagen fibers between the tendon and the surrounding bone was noted throughout the length of the bone tunnel, resembling a fibrous enthesis. Finally, tendon-to-bone healing was complete 3 to 6 months after the operation. In our study, the continuity of the repaired rotator cuff tendon was well-maintained despite the increase in the size of the peri-anchor cyst throughout the followup period. Thus, we assume that 3 months is enough time to complete the process of tendon-to-bone healing and initiation of peri-anchor (PLDLA) cyst formation after 3 months has little effect on rotator cuff tendon-to-bone healing.

Peri-anchor cyst formation at a PGA anchor, which was the first-generation anchor, could be problematic because the anchor degrades rapidly. On the other hand, second-generation anchors, like PLDLA anchors, allow more time for the tendonto-bone healing process since the degradation time is much longer. The final outcome of rotator cuff repair using these secondgeneration anchors appears to have little association with perianchor cyst formation. ${ }^{1)}$ However, in our case, peri-anchor cyst formation resulted in synovitis and effusion in the joint, which led to movement-related pain and the need for additional treatments including corticosteroid injection. Therefore, surgeons should be aware of the possibility of peri-anchor cyst formation and the complications related to bio-absorbable anchors. Surgeons should also consider other types of anchors composed of similar bio-composite materials in order to avoid postoperative peri-anchor cyst formation.

Peri-anchor (PLDLA) cyst formation can occur occasionally and induce glenohumeral joint synovitis and painful limited motion of shoulder joints as in the current case. Despite this, the integrity of the rotator cuff and the long-term clinical outcome were not affected by the formation of the peri-anchor cyst. Surgeons should be aware of the possibility of peri-anchor cyst formation and should monitor for large peri-anchor cyst formation after ARCR. Despite large peri-anchor cyst formation after ARCR, good long-term outcome can be achieved.

\section{References}

1. Kim SH, Oh JH, Lee OS, Lee HR, Hargens AR. Postoperative imaging of bioabsorbable anchors in rotator cuff repair. Am J Sports Med. 2014;42(3):552-7. doi: 10.1177/ 
0363546513517538.

2. Nho SJ, Provencher MT, Seroyer ST, Romeo AA. Bioabsorbable anchors in glenohumeral shoulder surgery. Arthroscopy. 2009;25(7):788-93. doi: 10.1016/j.arthro.2008.08.018.

3. Ozbaydar M, Elhassan B, Warner JJ. The use of anchors in shoulder surgery: a shift from metallic to bioabsorbable anchors. Arthroscopy. 2007;23(10):1124-6. doi: 10.1016/j.arthro. 2007.05.011.

4. Tan CK, Guisasola I, Machani B, et al. Arthroscopic stabilization of the shoulder: a prospective randomized study of absorbable versus nonabsorbable suture anchors. Arthroscopy. 2006;22(7):716-20. doi: 10.1016/j.arthro.2006.03.017.

5. Speer KP, Warren RF. Arthroscopic shoulder stabilization. A role for biodegradable materials. Clin Orthop Relat Res. 1993;(291):67-74.

6. Berg EE, Oglesby JW. Loosening of a biodegradable shoulder staple. J Shoulder Elbow Surg. 1996;5(1):76-8. doi: 10.1016/ S1058-2746(96)80035-8.
7. Saikku-Bäckström A, Tulamo RM, Räihä JE, et al. Intramedullary fixation of cortical bone osteotomies with absorbable selfreinforced fibrillated poly-96L/4D-lactide (SR-PLA96) rods in rabbits. Biomaterials. 2001;22(1):33-43. doi: 10.1016/S01429612(00)00142-3.

8. Macarini L, Murrone M, Marini S, Mocci A, Ettorre GC. [MRI in $\mathrm{ACL}$ reconstructive surgery with PDLLA bioabsorbable interference screws: evaluation of degradation and osteointegration processes of bioabsorbable screws]. Radiol Med. 2004;107(12):47-57. Italian.

9. Barber FA, Dockery WD. Long-term absorption of poly-L-lactic Acid interference screws. Arthroscopy. 2006;22(8):820-6. doi: 10.1016/j.arthro.2006.04.096.

10. Rodeo SA, Arnoczky SP, Torzilli PA, Hidaka C, Warren RF. Tendon-healing in a bone tunnel. A biomechanical and histological study in the dog. J Bone Joint Surg Am. 1993;75(12):1795803. doi: 10.2106/00004623-199312000-00009. 